\title{
Adiabatic partial Siberian snake turn-on with no beam depolarization
}

R. A. Phelps, V. A. Anferov ${ }^{(a)}$, C. M. Chu, E. D. Courant ${ }^{(b)}$, D. A. Crandell, Ya. S. Derbenev ${ }^{(c)}$, W. A. Kaufman, A. V. Koulsha ${ }^{(a)}$, A. D. Krisch,

T. S. Nurushev, D. B. Raczkowksi, S. E. Sund and V. K. Wong ${ }^{(d)}$

Randall Laboratory of Physics, University of Michigan, Ann Arbor, Michigan 48109-1120

D. D. Caussyn, T. J. P. Ellison, S. Y. Lee, F. Sperisen,

E. J. Stephenson and B. von Przewoski

Indiana University Cyclotron Facility, Bloomington, Indiana 47408-0768

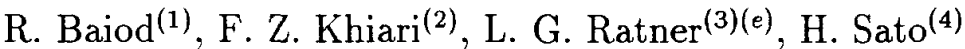

(1) Fermilab, Batavia, Illinois 60510

(2) Energy Research Laboratory, King Fahd University, Dhahran 31261, Saudi Arabia

(3) AGS Department, Brookhaven National Laboratory, Upton, New York 11973

(4) KEK, 1-1 Oho, Tsukuba-shi, Ibaraki-ken 305, Japan

(Received 17 December 1993)

A recent experiment in the IUCF cooler ring studied the adiabatic turn-on of a partial Siberian snake at $370 \mathrm{MeV}$, where the spin tune, $\nu_{s}$, is $2 \frac{1}{2}$ for all snake strengths. The snake consisted of two rampable warm solenoid magnets in series with a superconducting solenoid; this combination allowed varying the snake strength between about 0 and $25 \%$ at $370 \mathrm{MeV}$. We measured the beam polarization after varying the snake either once, twice or ten times; we found with good precision that no polarization was lost. This supports the conjecture that a Siberian snake can be ramped adiabatically at an energy where the spin tune is a half-integer.

PACS Numbers: 41.75.Ak, 07.77.+p, 29.27.Bd, 29.27.Hj

Acceleration of a polarized proton beam to high energy requires overcoming the many spin-depolarizing resonances that occur in circular accelerators. The individual resonance correction technique was quite successful at the ZGS [1], Saturne [2], KEK [3] and the AGS [4]; however, this technique becomes impractical above beam energies of about $20 \mathrm{GeV}$. Fortunately, recent experiments [5-8] demonstrated the ef- 
fectiveness of the Siberian snake technique [9], which should simultaneously overcome all depolarizing resonances at any energy. A Siberian snake forces all depolarizing effects to cancel themselves by rotating each proton's spin by $180^{\circ}$ on each turn around an accelerator ring. This spin rotation can be produced by using either a solenoid magnet, which has a longitudinal field, or a combination of transverse field dipole magnets, which only distorts the beam orbit inside the snake itself. A transverse Siberian snake is especially effective at energies above $100 \mathrm{GeV}$. However, at lower energies the orbit excursions inside a transverse snake become rather large; this increases the transverse size and cost of the snake dipoles. At higher energies the Lorentz transformation requires very long solenoids; at $20 \mathrm{TeV}$ a $180^{\circ}$ spin rotation requires a $9 \mathrm{~T}$ solenoid about $10 \mathrm{~km}$ long. Moreover, at all energies a solenoid snake focuses the beam and produces strong coupling between the vertical and horizontal betatron motion.

These orbit problems are especially serious near low-energy injection in mediumenergy accelerators such as the Fermilab Main Injector [10], the SSC Medium Energy Booster [11], and the Brookhaven RHIC [12]. However, these accelerators need fullstrength Siberian snakes to overcome the many strong depolarizing resonances near the top energy of their acceleration cycle. Therefore, we experimentally studied the adiabatic turn-on of a Siberian snake. If adiabatic turn-on causes no depolarization, then it could be used to simultaneously solve both the depolarization problem and the orbit problem. The strong higher energy depolarizing resonances could be overcome by adiabatically turning-on a full snake at about $20 \mathrm{GeV}$; the weaker low energy resonances could then be overcome using other techniques $[4,10]$, which do not cause large orbit excursions at low energy.

In a circular accelerator ring with no Siberian snakes, each proton's spin precesses around the vertical field of the accelerator's dipole magnets; however, any horizontal magnetic fields can depolarize the beam. This depolarization occurs when the spin precession frequency, $f_{s}$, satisfies the resonance condition

$$
f_{s}=f_{c} \nu_{s}=f_{c}\left(n+m \nu_{y}\right) \text {, }
$$

where $n$ and $m$ are integers; $f_{c}$ is the protons' circulation frequency; the vertical betatron tune, $\nu_{y}$, is the number of vertical betatron oscillations during each turn around the ring; and the spin tune, $\nu_{s}$, is the number of spin precessions during each turn around the ring. The imperfection resonances occur when $\mathrm{m}=0$, while the first-order intrinsic resonances occur when $\mathrm{m}= \pm 1$.

With no Siberian snake, the spin tune is proportional to the proton's energy

$$
\nu_{s}=G \gamma,
$$

where $\gamma$ is the Lorentz energy factor and $G=1.792847$ is the proton's anomalous magnetic moment. A recent experiment [7] confirmed that in a ring containing a partial Siberian snake of strength $s$, the spin tune obeys the equation 


$$
\cos \left(\pi \nu_{s}\right)=\cos (\pi G \gamma) \cos \left(\frac{\pi s}{2}\right)
$$

where $s=1$ corresponds to a full snake, which rotates the spin by $180^{\circ}$; this behavior of the spin tune versus the snake strength is shown in Fig. 1 for different beam energies. For a full Siberian snake, the spin tune is equal to a half-integer at all energies. For any snake strength, Eq. 3 indicates that the spin tune is equal to a half-integer whenever the kinetic energy, $\mathrm{T}$, satisfies

$$
T=370 \mathrm{MeV}+k 523 \mathrm{MeV},
$$

where $k$ is an integer. These "half-integer" energies certainly seem best for changing the snake strength; at other energies the spin tune would change along with the snake strength and might cross depolarizing resonances.

The $2 \mathrm{~T} \cdot \mathrm{m}$ superconducting Siberian snake solenoid, the polarimeter and the Cooler Ring's operation with polarized protons were discussed earlier $[5-8,13]$. To allow us to adiabatically vary a Siberian snake's strength, we recently built two rampable warm $0.2 \mathrm{~T} \cdot \mathrm{m}$ solenoids of 1026 turns each. These warm solenoids were placed in series with our superconducting snake solenoid as shown in Fig. 2. The snake strength, $s$, for a solenoid magnet of $N I$ ampere turns is given by

$$
s=\frac{\mu_{0}(1+G)}{10.479 p} N I
$$

where $\mu_{0}=4 \pi 10^{-7} \mathrm{~T} \cdot \mathrm{m} \mathrm{A} \mathrm{A}^{-1}$ and $p$ is the proton's momentum in $\mathrm{GeV} / \mathrm{c}$. The superconducting solenoid was operated at $37.1 \mathrm{~A}$, which made it about a $12.5 \%$ snake at $370 \mathrm{MeV}$ using the earlier calibration [14].

The polarized proton beam was then injected into the Cooler Ring at $200 \mathrm{MeV}$ which is well above the $G \gamma=2$ imperfection depolarizing resonance [5] near $108 \mathrm{MeV}$ and the $G \gamma=7-\nu_{y}$ intrinsic depolarizing resonance [6] near $177 \mathrm{MeV}$. The beam was next accelerated to $370 \mathrm{MeV}$ with the superconducting solenoid current fixed at 37.1 A. During this acceleration the superconducting solenoid decreased from being about an $18 \%$ snake at $200 \mathrm{MeV}$ to about a $12.5 \%$ snake at $370 \mathrm{MeV}$. After the beam reached $370 \mathrm{MeV}$, the two warm solenoid magnets varied together from 0 to $-165 \mathrm{~A}$ each, which is about a $-6.2 \%$ snake each; then they varied together in the opposite direction to become about a $+6.2 \%$ snake each. As shown in Fig. 3, this process varied the total snake strength of the three solenoids between about $0 \%$ and $25 \%$.

For a Siberian snake turn-on to be adiabatic, the stable spin direction must change much more slowly than the time for a proton to circle the ring; thus the snake turn-on time $\Delta t$ must satisfy the relation

$$
\Delta t \gg \frac{1}{f_{c}}
$$


This adiabatic requirement was certainly satisfied for our $1 \mathrm{~s}$ ramp time shown in Fig. 3 ; the $\Delta t$ was about $10^{6}$ times slower than the inverse of the protons' circulation frequency $f_{c}$ which was $2.4 \mathrm{MHz}$ at $370 \mathrm{MeV}$.

The transverse beam polarization, $P_{t}=\sqrt{P_{v}^{2}+P_{r}^{2}}$, is plotted against the number of adiabatic turn-ons and turn-offs in Fig. 4. With a $0 \%$ snake, the stable spin direction was vertical; the $25 \%$ partial Siberian snake tilted the stable spin direction by about $23^{\circ}[15]$. Once the beam reached $370 \mathrm{MeV}$, where the spin tune was $2 \frac{1}{2}$, there was no depolarization as we turned the $25 \%$ partial Siberian snake on and off. As shown in Fig. 4, the beam polarization measured after either one, two, or ten adiabatic snake changes was exactly the same, within our precision of about $2 \%$.

This experimental data supports the conjecture that the strength of a Siberian snake can be varied adiabatically without depolarizing the beam at an energy where the spin tune is a half-integer. Thus, the proton's spin precession axis appears to follow the motion of the stable spin direction during the adiabatic turn-on or turnoff of a partial Siberian snake. This adiabatic Siberian snake turn-on capability could allow much more efficient acceleration of polarized proton beams at mediumenergy accelerators such as the Fermilab Main Injector [10], the SSC Medium Energy Booster [11], or the Brookhaven RHIC [12].

We would like to thank J. M. Cameron and the entire Indiana University Cyclotron Facility staff for the most successful operation of the Cooler Ring. We are grateful to A. W. Chao, S. V. Gladysheva, R. S. Herdman, S. Hiramatsu, M. Ho, H.-O. Meyer, M. G. Minty, J. B. Muldavin, C. Ohmori, R. E. Pollock, T. Rinckel, T. Roser, P. Schwandt, D. S. Shoumkin, T. Toyama, S. J. Wheeler, and K. White for their help with parts of this experiment. This research was supported by grants from the U.S. Department of Energy and the U.S. National Science Foundation. 


\section{REFERENCES}

(a) Also at: Moscow State University, Moscow, Russia.

(b) Also at: Brookhaven National Laboratory.

(c) Also at: Department of Nuclear Engineering, University of Michigan.

(d) Also at: Office of the Provost, University of Michigan at Flint.

(e) Present Address: Department of Physics, University of Michigan.

[1] T. Khoe et al., Part. Accel. 6, 213 (1975).

[2] J. L. Laclare et al., J. Phys. (Paris), Colloq. 46, C2-499 (1985).

[3] H. Sato et al., Nucl. Instrum. Methods Phys. Res. Sect. A 272, 617 (1988).

[4] F. Z. Khiari et al., Phys. Rev. D39, 45 (1989).

[5] A. D. Krisch et al., Phys. Rev. Lett. 63, 1137 (1989).

[6] J. E. Goodwin et al., Phys. Rev. Lett. 64, 2779 (1990).

[7] V. A. Anferov et al., Phys. Rev. A46, R7383 (1992).

[8] R. Baiod et al., Phys. Rev. Lett. 70, 2557 (1993).

[9] Ya. S. Derbenev and A. M. Kondratenko, Part. Accel. 8, 115 (1978).

[10] Acceleration of Polarized Protons to 120 and $150 \mathrm{GeV}$ in the Fermilab Main Injector, SPIN Collaboration, Univ. of Michigan Report (March 1992), (unpublished).

[11] Proceed. 1985 Ann Arbor Workshop on Polarized Beams at SSC, AIP Conf. Proc. 145, eds. A. D. Krisch, A. M. T. Lin, O. Chamberlain (AIP, New York 1986).

[12] RHIC Spin Proposal M. Beddo et al.; Brookhaven National Lab proposal (1992), (unpublished).

[13] M. G. Minty et al., Phys. Rev. D44, R1361 (1991).

[14] J. E. Goodwin, Ph.D. Thesis, Indiana University (1990).

[15] Two solenoids in the injection beam line allowed adjustment of the injected beam's polarization direction to match the Cooler Ring's stable spin direction at $200 \mathrm{MeV}$ injection with the partial Siberian snake. However the match was probably not exact. Thus, there may have been a few per cent polarization loss at injection. 


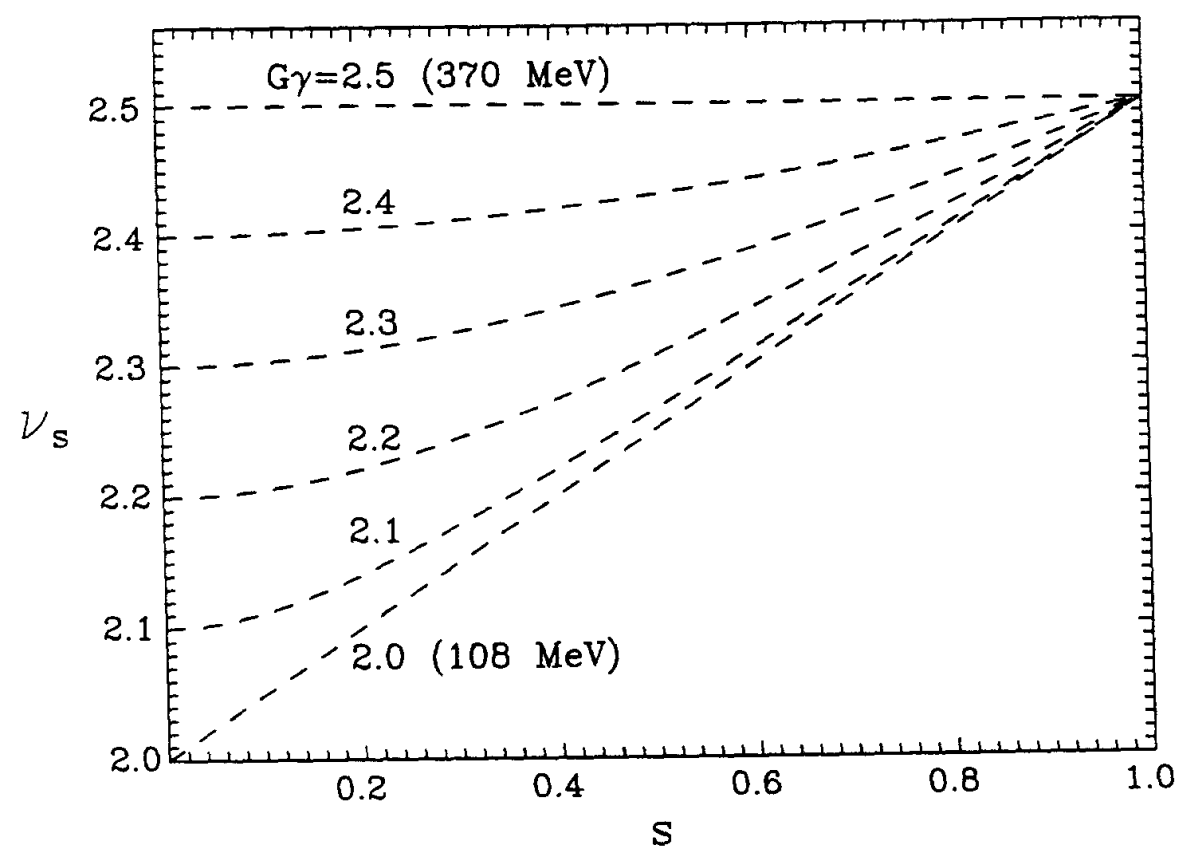

FIG. 1. The spin tune, $\nu_{s}$, is plotted against the snake strength, $s$, for different values of $\mathrm{G} \gamma$ using Eq. 3 .

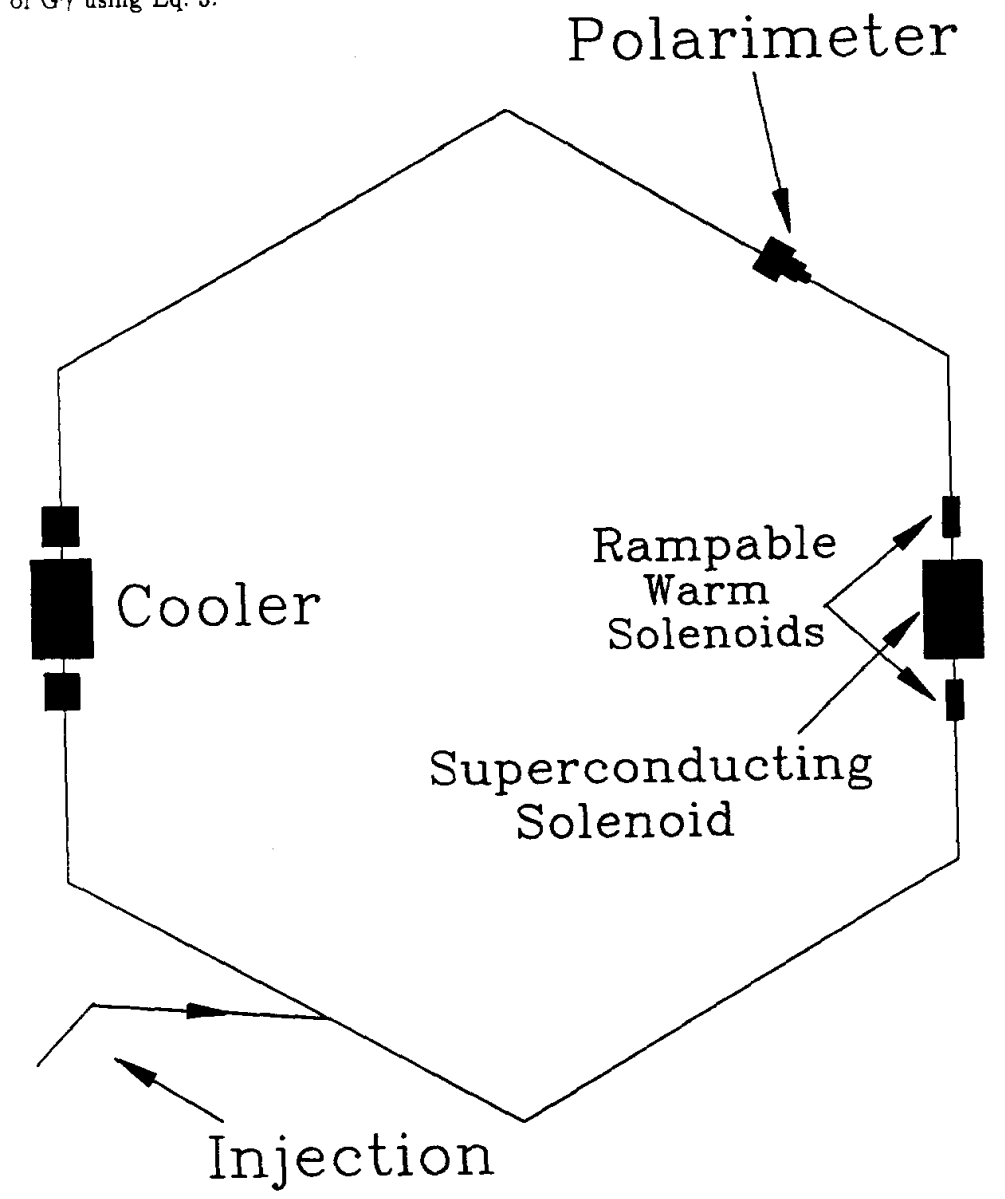

FIG. 2. Location of the two warm rampable solenoids, the superconducting solenoid, and other relevant hardware in the IUCF Cooler Ring. 


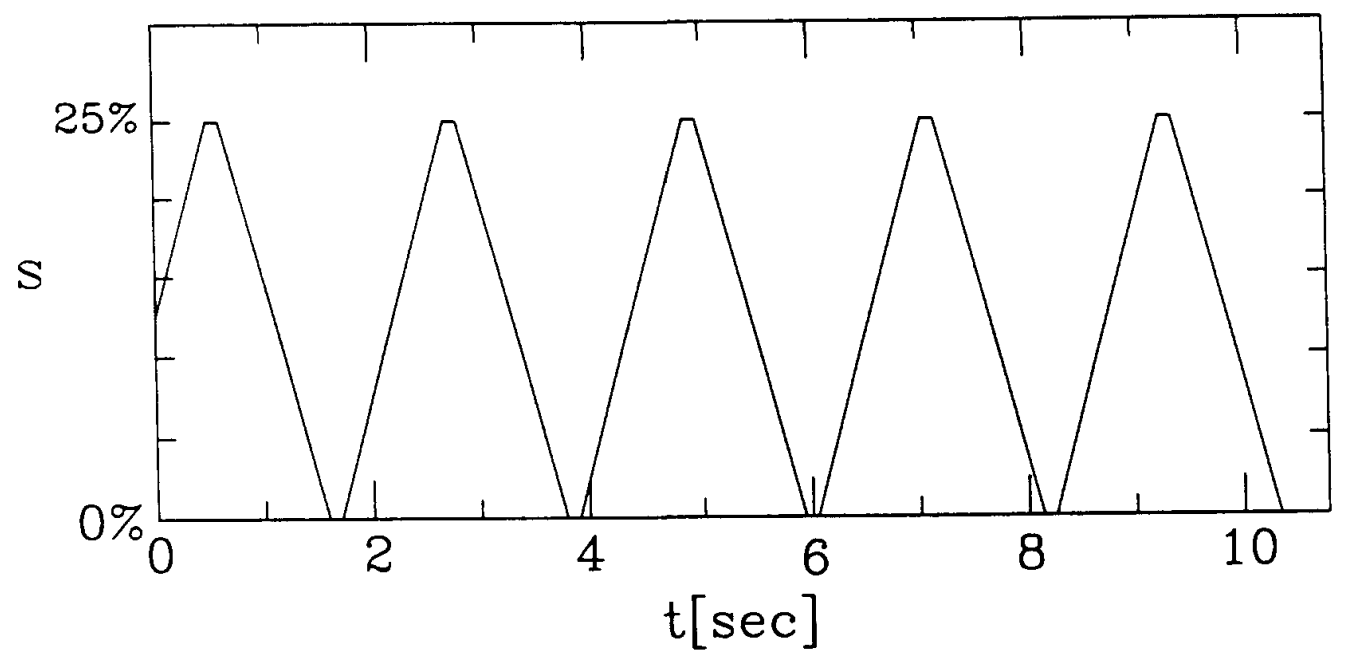

FIG. 3. The waveform for the Siberian snake ramp. The superconducting solenoid current was fixed at $37.1 \mathrm{~A}$, which was about $12.5 \%$ of a full snake at $370 \mathrm{MeV}$, while the two warm solenoids were varied together up and down from jointly forming a $-12.5 \%$ snake $(-165 \mathrm{~A}$ each $)$ to $\mathrm{a}+12.5 \%$ snake. Thus, the total snake varied repeatedly between 0 and $25 \%$.

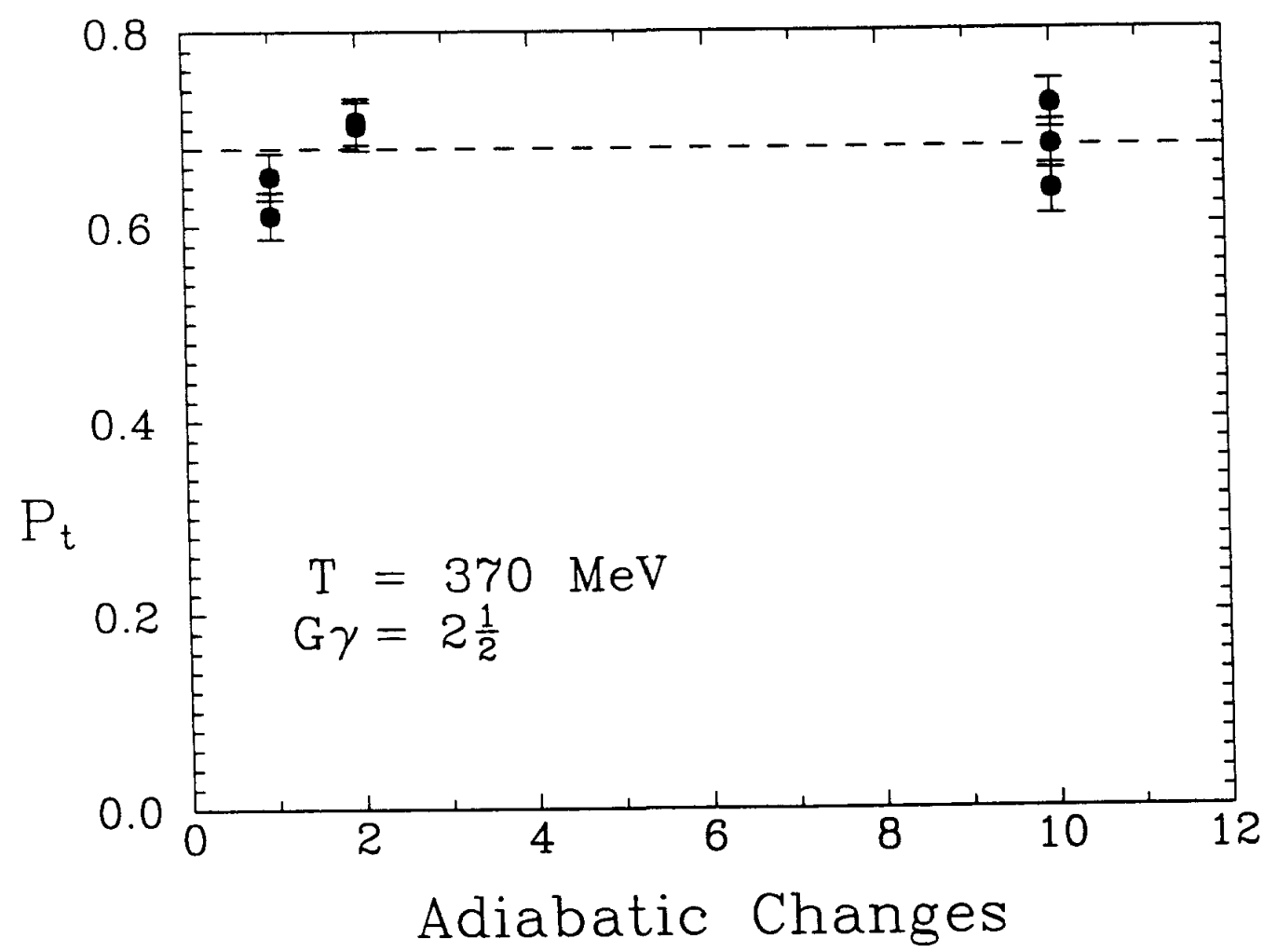

FIG. 4. The transverse polarization, $P_{t}=\sqrt{P_{v}^{2}+P_{\tau}^{2}}$, at $370 \mathrm{MeV}$ is plotted against the number of times the $25 \%$ partial Siberian snake was turned on or off. The dashed line is the best fit to the data; the data shows no depolarization within our $2 \%$ precision. 
